\title{
Atelocollagen Enhanced Osteogenesis in a Geometric Structured Beta-TCP Scaffold by VEGF Induction
}

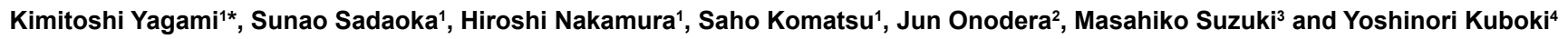

${ }^{1}$ Division of Oral Health Promotion, Institute for Oral Science, Matsumoto Dental University, Shiojiri, Japan

${ }^{2}$ Koken Co., Ltd., Tokyo, Japan

${ }^{3}$ Pilot Co., Ltd., Tokyo, Japan

${ }^{4}$ Graduate School of Environmental Earth Science, Hokkaido University, Sapporo, Japan

\begin{abstract}
In order to establish the convertibility of a host for bone augmentation, we herein developed a new honeycombshaped $\beta$-tricalcium phosphate $(37 \mathrm{H})$ using atelocollagen as a scaffold, which exhibited unique geometric properties for in vitro and in vivo studies.

Human mesenchymal stem cells (MSC) were cultured with $37 \mathrm{H}$ or atelocollagen-coated honeycomb-shaped $\beta$-tricalcium phosphate $(\mathrm{Col} 37 \mathrm{H})$, and their osteoblastic differentiation was then analyzed. Atelocollagen promoted cell adhesion and formation of vessel-like structures in the tunnels of scaffolds of cultured MSC. The mRNA expression levels of type I collagen, osteocalcin, and VEGF were greater in MSC cultured with Col37H than with $37 \mathrm{H}$. Bone generation with Col37H in the rat calvaria was greater than with $37 \mathrm{H}$, and this was attributed to early vessel construction. A large number of blood vessels invaded tunnels from the periosteum and existing bone surface. A strong VEGF signal was detected immediately before the new bone surface in the tunnels of Col37H.
\end{abstract}

These results indicate that the addition of atelocollagen to $\mathrm{Col} 37 \mathrm{H}$ has potential in the construction of functional artificial bone.

Keywords: Honeycomb-shaped $\beta$-TCP; Atelocollagen; VEGF; Bone marrow mesenchymal stem cells; Blood vessels; Bone regeneration

\section{Introduction}

The early replacement of host bone with bone-filling materials is essential in bone regeneration therapy. The development of Harversian canals and angiogenesis are necessary to maintain normal bone metabolism in biomaterials at the site of bone regeneration, so that metabolism continues in the bone that forms, thereby contributing to its maintenance. Various bone-filling materials and bone formationaccelerating agents have been developed and successfully applied to clinical settings [1]. However, the histological structure of bone regenerated using current filling materials often differs from that by normal bone metabolism [2]. In the beginning of osteogenesis, the extracellular matrix proteins, such as collagen, gives an optimal environment structure for cell proliferation and differentiation to histogenesis following cell aggregation. Furthermore, some difficulties associated with complete treatments for bone defects have not yet been overcome. Delay of normal bone remodeling due to HA, premature absorption of collagen and TCP [3], and difficulty of controlling the regeneration volume with platelet-rich plasma (PRP) [4] and bone morphogenic protein-2 (BMP-2) [5] have been reported. These cytokines have been used in combination with scaffolds in order to stimulate the differentiation of a certain number of immature cells present in the bone microenvironment in the local tissues surrounding bone. However, controlling the quantity of cells and their activities at local sites is challenging, which may result in adverse effects in patients, and these cytokines are also expensive.

Previous findings [6-8] prompted us to consider the importance of an effective artificial extracellular matrix (ECM) in the induction of bone. Matrices have been categorized according to their (a) physical, (b) chemical, and (c) biochemical properties. A fourth property, (d) geometry, has also been proposed [9]. The superiority of honeycombshaped structures with numerous straight tunnels with diameters of 0.3-0.4 mm made from hydroxyapatite ceramics and collagenous preparations has indicated the suitability of this geometry for bone formation. We previously reported that less bone was generated in the absence of a cell transfer scaffold with an appropriate geometric structure [8]. Bone must form in a uniform manner, and achieving this represents a challenge in the field of biological tissue engineering.

Supply of cytokines to osteoblastic progenitor cells by neovascularization is important for satisfactory bone formation. Vascular endothelial growth factor (VEGF) has recently been shown to regulate perichondrial vascularity and early stage osteoblast differentiation in bone development [10]. This study demonstrated that progenitor-derived VEGF was required for blood vessel recruitment in the differentiation of osteoblast precursors in mice. Ogose et al. [11] showed that $\beta$-TCP scaffolds promoted early resorption by osteoclasts, the vascular invasion of macropores, and osteoblastic cell attachment on the surface on ceramic surfaces. They also detected reticular collagen fibrils in $\beta$-TCP micropore structures, which were directly connected to lamellar bone.

In order to determine the effects between angiogenesis and type I collagen during osteogenesis, we herein devised a new artificial ECM consisting of H- $\beta$-TCP with atelocollagen, and predicted that the tunnel

${ }^{*}$ Corresponding author: Kimitoshi Yagami, Division of Oral Health Promotion Institute for Oral Science, Matsumoto Dental University, 1780 Hirohoka Gohobara, Shiojiri, Nagano, 399-0781, Japan, Tel: +81-0263-51-2153; E-mail: kyagami@po.mdu.ac.jp

Received November 25, 2015; Accepted January 11, 2016; Published January 18, 2016

Citation: Yagami K Sadaoka S, Nakamura $\mathrm{H}$, Komatsu S, Onodera J, et at. (2016) Atelocollagen Enhanced Osteogenesis in a Geometric Structured BetaTCP Scaffold by VEGF Induction. J Tissue Sci Eng 7: 162. doi:10.4172/21577552.1000162

Copyright: @ 2016 Yagami K, et al. This is an open-access article distributed under the terms of the Creative Commons Attribution License, which permits unrestricted use, distribution, and reproduction in any medium, provided the original author and source are credited. 
Citation: Yagami K, Sadaoka S, Nakamura H, Komatsu S, Onodera J, et al. (2016) Atelocollagen Enhanced Osteogenesis in a Geometric Structured Beta-TCP Scaffold by VEGF Induction. J Tissue Sci Eng 7: 162. doi:10.4172/2157-7552.1000162

structure in this scaffold may guide the initial stages of bone formation in vitro and ex vivo.

\section{Materials and Methods}

\section{Preparation of scaffolds}

Honeycomb-shaped beta-tricalcium phosphate $(37 \mathrm{H}$ : height: 1.0 $\mathrm{mm}$, outer diameter: $3.0 \mathrm{~mm}$, inner diameter: $300 \mu \mathrm{m})$ was provided for this study by Pilot Corporation (Tokyo, Japan) [12] by pushing out molding, and burned at 1,050 degrees. Atelocollagen-coated honeycomb-shaped beta-tricalcium phosphate discs $(\mathrm{Col} 37 \mathrm{H})$ were provided by Koken Co., LTD. (AteloCelloRR, Tokyo, Japan) (Figure 1). As for the $\mathrm{Col} 37 \mathrm{H}$ preparation, $37 \mathrm{H}$ was rinsed with ethanol/acetone (1:1) three times and was dried after washing three times in $\mathrm{H} 2 \mathrm{O}$ for five minutes. And then, it was sterilized by gamma beam after collagen crosslinking by UV irradiation (UV Crosslinker, CL-1000, UVP inc., CA, USA).

\section{MSC cultures with scaffolds}

Human mesenchymal stem cells (MSC) were purchased commercially (Lonza Co., Ltd, Visp, Switzerland). These cells were cultured in mesenchymal stem cell basal medium (MSCBM) with mesenchymal cell growth supplement (MCGS), according to the

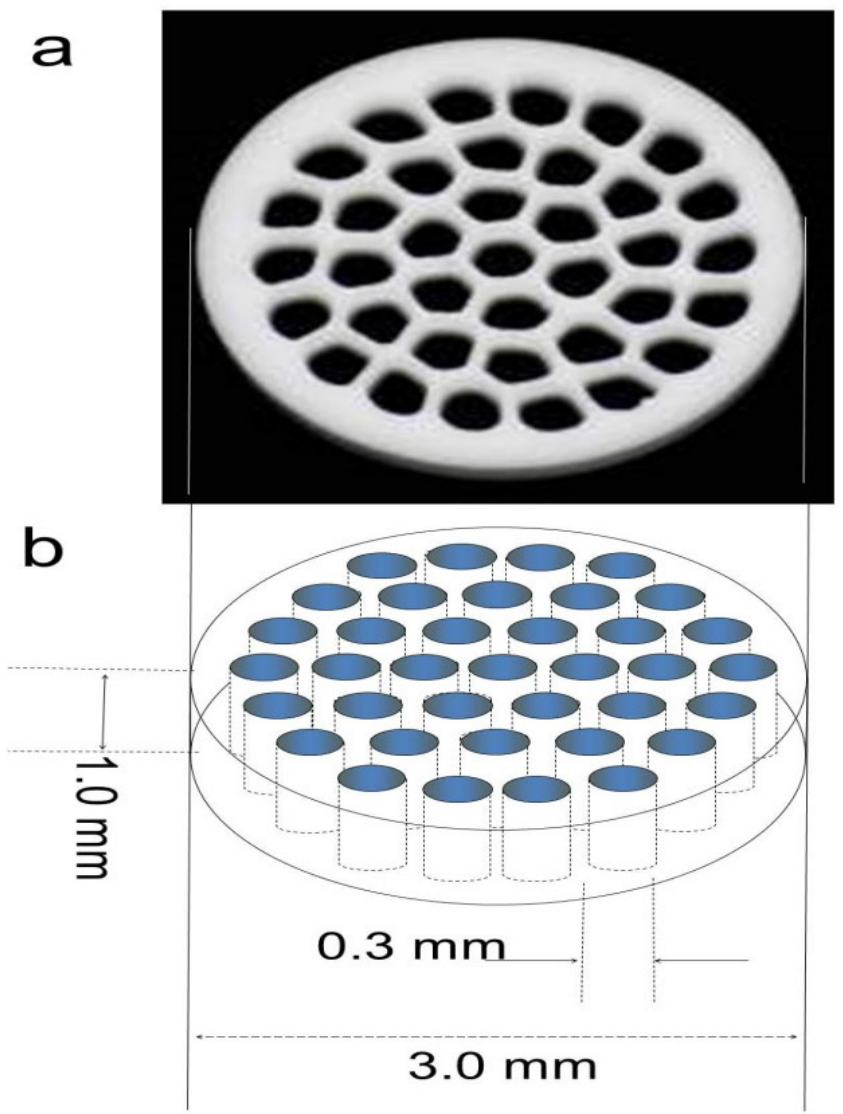

Figure 1: Photomicrogram (A) and structure (B) of honeycomb-shaped betatricalcium phosphate with or without atelocollagen. Scaffolds had 37 paralle tunnels that made the honeycomb shape. $37 \mathrm{H}$ was prepared with a special nozzle device as previously described [6]. A paste of hydroxyapatite powder was extruded through a multi-nozzled disc, in the form of multiple rods, into a condensation space of taped conical tubing. The cylindrical products were sintered at $1,000^{\circ} \mathrm{C}$ and cut to the required lengths. manufacturer's protocol. The medium in the flask was replaced every other day with the same volume of fresh medium.

In order to investigate the osteogenic differentiation of MSC, $5 \times 10^{5}$ cells $/ \mathrm{cm}^{2}$ were combined with $37 \mathrm{H}$ or $\mathrm{Col} 37 \mathrm{H}$ in osteogenic medium (Lonza Walkersville Inc., MD, USA) and cultured according to the manufacturer's protocol. After each differentiation culture period, the cells were fixed and stained with alkaline phosphatase (APase) using previously described methods [13]. After the indicated culture periods, three parallel sets of cell samples were employed to determine APase activities using histochemical and gene expression analyses.

\section{Immunocytochemistry, APase activity, and extracellular matrix synthesis}

The expression of type I collagen by hMSC cells on each scaffold was assessed using indirect immunostaining by a streptavidinperoxidase system (Nichirei, Tokyo, Japan) with an anti-type I collagen antibody or anti-vascular endothelial growth factor (VEGF) (Abcam, Cambridge, UK). After the experiment, cells were rinsed with PBS and fixed with $4 \%$ paraformaldehyde at $4^{\circ} \mathrm{C}$ for 15 minutes. The antibody signal was detected using a peroxidase anti-fluorescein detection kit (Vector laboratories Inc., Burlingame, CA, U.S.A). In order to detect APase activity, fixed samples were rinsed twice in saline and incubated in $50 \mathrm{mM}$ Tris- $\mathrm{HCl}$ (pH 9.5) containing $0.5 \mathrm{mg} / \mathrm{ml}$ of naphthol AS-BI phosphate (Sigma, St. Louis, USA) and $1 \mathrm{mg} / \mathrm{ml}$ of fast red trisodium salt (Sigma, St. Louis, USA) at $37^{\circ} \mathrm{C}$ for $20 \mathrm{~min}$. All cell nuclei were stained with 4', 6-diamidino-2-phenylindole (DAPI) (Vector Laboratories Inc., Burlingame, CA, U.S.A.).

\section{Measurement of APase activity}

APase activity was also determined biochemically after the solubilization of cells with $1.5 \mathrm{M}$ Tris buffer containing $1 \%$ Triton $\mathrm{X}-100$, as described previously [13]. The substrate used was $5 \mathrm{mM}$ $p$-nitrophenylphosphate (Sigma, St. Louis, MO) and activity was expressed as nmol of $p$-nitrophenol $(p-\mathrm{NP})$ formed $/ \mathrm{min} / \mu \mathrm{g}$ protein assuming $1 \mathrm{~A}_{410}=64 \mathrm{nmol}$ of the product. Protein levels were determined using the Lowry protein assay (Bio-Rad Laboratories, Inc., Tokyo, Japan).

\section{Quantitative RT-PCR analysis}

The expression of type I collagen, osteocalcin, and VEGF mRNA in MSC was determined by RT-PCR (TaqMan Gene Expression Assays, Thermo Fisher Scientific, USA). Total RNA was isolated from the H- $\beta$-TCP/MSC complex using One-Step Real-Time RTPCR Master Mixes ' (Invitrogen, USA), followed by an amplification step using the Single Cell-to- $\mathrm{C}_{\mathrm{T}}{ }^{\mathrm{m}} \mathrm{Kit}{ }^{\circ}$ (Life Technologies, USA). PCR amplification was performed using sense- and anti-sense-specific primers for human osteocalcin (the bone gamma-carboxyglutamate (gla) protein: Hs01587814_g1), vascular endothelial growth factor (VEGF: Hs00900055_m1), type I collagen (collagen, type I, alpha 1: Hs00164004_ml), and glyceraldehyde-3-phosphate dehydrogenase (GAPDH: Hs02758991_g1) according to the manufacturer's protocol.

\section{Transplantation of scaffolds and in vivo conditions}

Thirty six Sprague Dawley rats (six weeks old, male), each weighing between 160 and $180 \mathrm{~g}$ (Japan SLC, Shizuoka, Japan), were fed a normal diet in an optimal environment. The animals were randomly allocated into 1- to 6-week groups with 6 rats in each group. All animal procedures were performed in accordance with The Guidelines of The Ministry of Education, Culture, Sports, Science and Technology 
Citation: Yagami K, Sadaoka S, Nakamura H, Komatsu S, Onodera J, et al. (2016) Atelocollagen Enhanced Osteogenesis in a Geometric Structured Beta-TCP Scaffold by VEGF Induction. J Tissue Sci Eng 7: 162. doi:10.4172/2157-7552.1000162

Page 3 of 8

Japan. All experiments using rats were approved by the Matsumoto Dental University Experimental Animal Committee and conducted in accordance with their guidelines for studies with laboratory animals for the accomplishment of $3 \mathrm{Rs}$. $37 \mathrm{H}$ or $\mathrm{Col} 37 \mathrm{H}$ was transplanted onto the rat calvaria, as shown in Figures $2 \mathrm{~A}$ and $2 \mathrm{~B}$, under general anesthesia. Calcein blue (day 0$)$ and alizarin complexion $(24 \mathrm{~h}$ before each experimental period) were injected subcutaneously for labeling bone generation. Animals were sacrificed under general anesthesia 1 to 6 weeks after implantation. Scaffolds were removed with the surrounding soft tissues and bone and then fixed with $10 \%$ neutral-buffered formalin at $4^{\circ} \mathrm{C}$ for 72 hours.

\section{Peripheral quantitative computed tomography analysis}

Before histological analyses were conducted, all $37 \mathrm{H}$ and $\mathrm{Col} 37 \mathrm{H}$ samples were analyzed using peripheral quantitative computed tomography (pQCT: XCT Research SA+, Stratec Medizintechnik $\mathrm{GmbH}$, Pforzheim, Germany) to evaluate bone mineral density

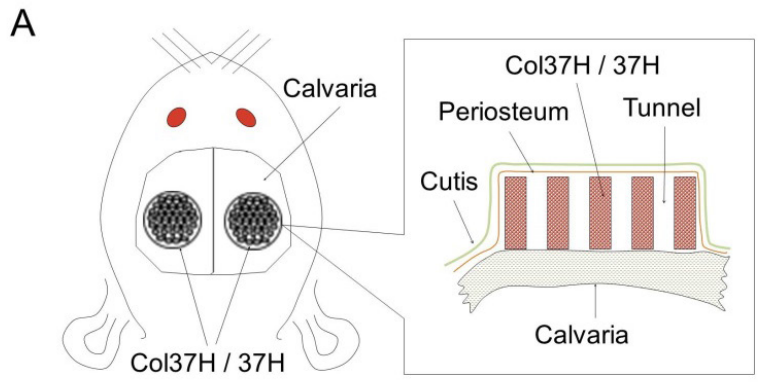

B
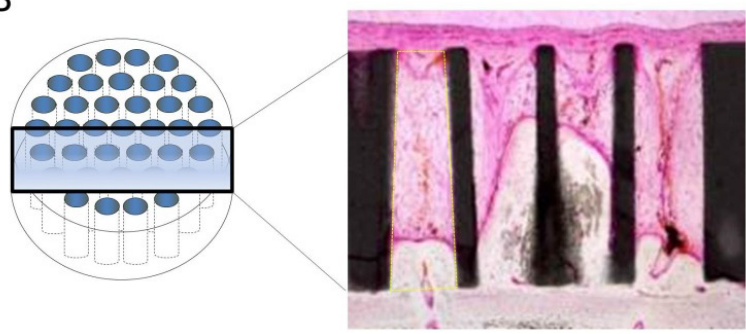

C

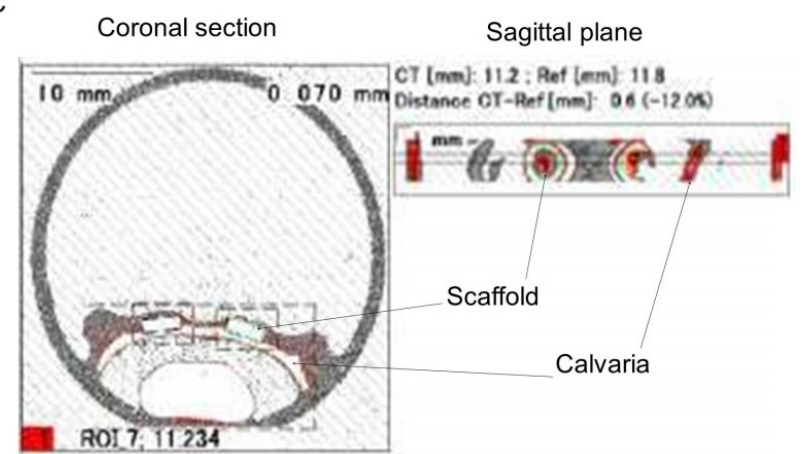

Figure 2: $37 \mathrm{H}$ and $\mathrm{Col} 37 \mathrm{H}$ were applied symmetrically and horizontally as a pair to the surface of the calvaria and covered with periosteum $(A$, Insert shows a cross-sectional drawing of the setting). Design of the analysis for bone mineral density and histological findings in the tunnels of the scaffolds (B). XCT image of ROI (Yellow square, $3.0 \times 1.0 \mathrm{~mm}$, top left panel), a sliced image (top middle panel), and slice thickness (top right panel, $1.0 \mathrm{~mm})(\mathrm{C})$. Details of the $\mathrm{ROI}$ area for total bone density (bottom left), trabecular density (bottom left), and cortical density (bottom right).
(BMD), according to the manufacturer's protocol. Fixed samples were prepared for a coronal section of scaffold tunnels on XCT (Figure 2C). Analyses included BMD measurements of two regions: cortical bone (the $\beta$-TCP area of $37 \mathrm{H}$ or $\mathrm{Col} 37 \mathrm{H}$ ) and trabecular bone (the tunnel area of $37 \mathrm{H}$ or $\mathrm{Col} 37 \mathrm{H}$ ), as shown in Figure 2C. The area of ROI for each measurement was determined to be $3 \mathrm{~mm}^{3}$ (width $3.0 \mathrm{~mm} \times$ height $1.0 \mathrm{~mm} \times$ thickness $1.0 \mathrm{~mm}$ ) with total bone density (TB). The CT density values measured reflected the summed effects of new bone and $\beta$-TCP.

\section{Histological analysis}

Tissue samples were embedded in polyester resin (Technovit 8100 Heraeus Kulzer, Germany) to prepare undecalcified sections according to the manufacturer's protocol. Three- $\mu$ m-thick sections along the tunnel of the scaffold were prepared. Sections were stained with an antiVEGF antibody and Villanueva osteochrome bone stain (Polysciences, Inc., Warrington, PA, USA).
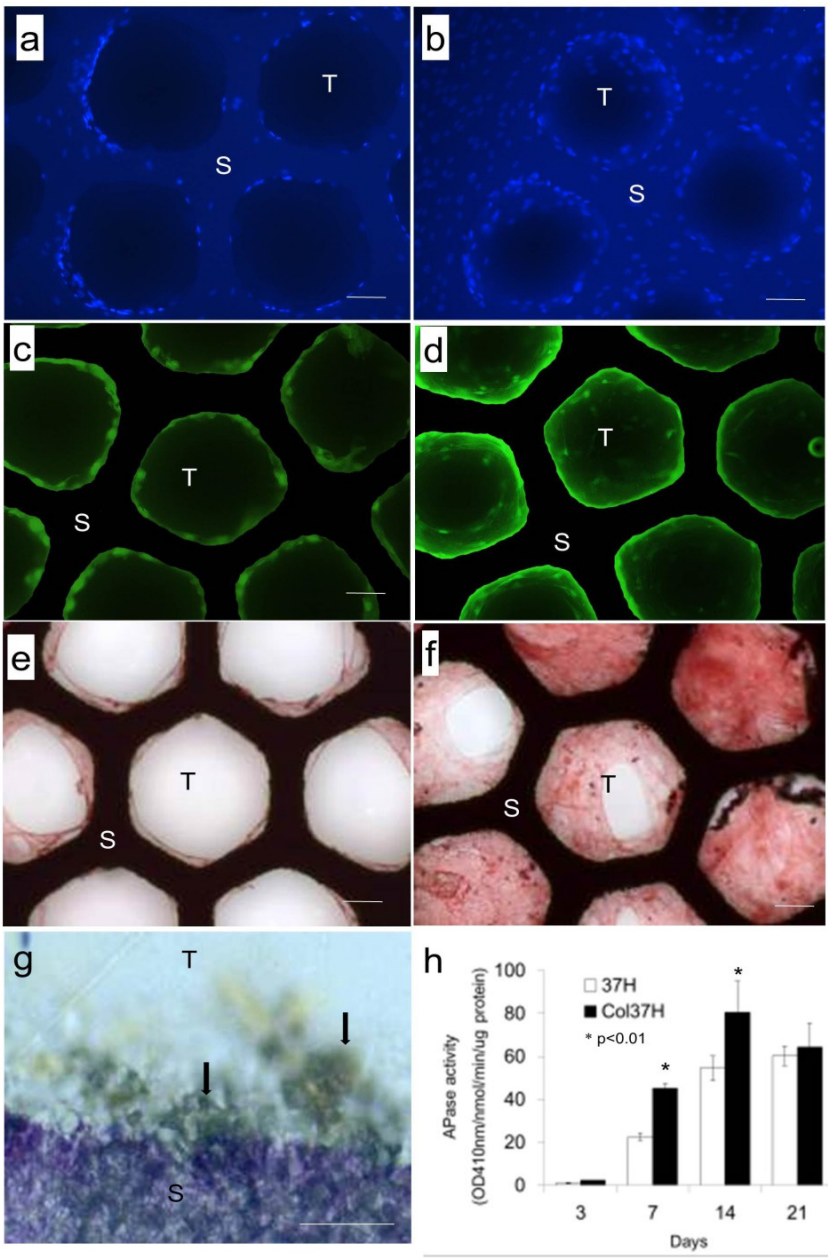

Figure 3: Osteogenic induction of MSC on $\mathrm{Col} 37 \mathrm{H}$ and $37 \mathrm{H}$.

Microscopic findings of MSC cultured on $37 \mathrm{H}(\mathrm{a}, \mathrm{c}, \mathrm{e})$ and $\mathrm{Col} 37 \mathrm{H}(\mathrm{b}, \mathrm{d}, \mathrm{f})$ Samples were stained with DAPI ( $a$ and $b$ ), type I collagen (c and d), VEGF (e and f) on day 7 , and APase (g) on day 14 of the culture. Bars, a-e: $100 \mu \mathrm{m}$, $\mathrm{g}: 20 \mu \mathrm{m}$. The APase-stained cell layer on the inside of the tunnel of $\mathrm{Col} 37 \mathrm{H}$ (g, arrows). APase activities were determined on days 3, 7, 14, and 21 of the culture with $37 \mathrm{H}(\square)$ or $\mathrm{Col} 37 \mathrm{H}(\mathbf{\square})$ (h). Values represent means $\pm \mathrm{SE}$ of 6 sets of cultures. Each significant difference was tested between $37 \mathrm{H}$ and $\mathrm{Col} 37 \mathrm{H}$ at each time point $\left({ }^{*} p<0.01\right)(\mathrm{h})$. S: Scaffold, T: Tunnel. 
Citation: Yagami K, Sadaoka S, Nakamura H, Komatsu S, Onodera J, et al. (2016) Atelocollagen Enhanced Osteogenesis in a Geometric Structured Beta-TCP Scaffold by VEGF Induction. J Tissue Sci Eng 7: 162. doi:10.4172/2157-7552.1000162

Page 4 of 8

\section{Statistical analysis}

Unless otherwise noted, MSC from each of the rats were characterized using the assays described above, and representative data sets are shown. Quantitative results are presented as the mean \pm
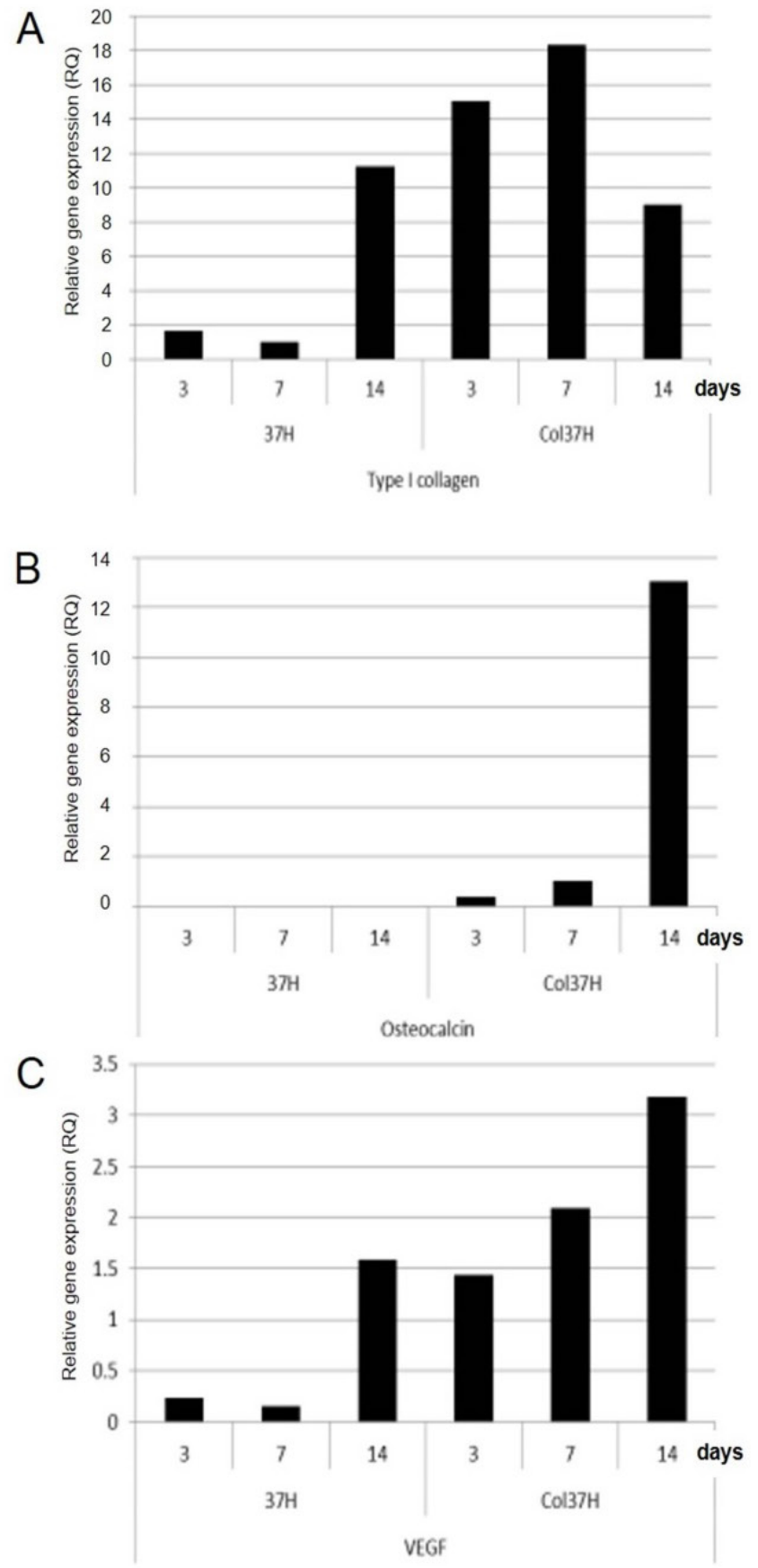

Figure 4: Comparison between osteogenic gene expression on MSC in $\mathrm{Col} 37 \mathrm{H}$ and $37 \mathrm{H}$. Total RNA was extracted from cells that adhered to a scaffold after the culture with Col37H and $37 \mathrm{H}$ in $\mathrm{hMSC}$ for 3,7 , and 14 days $(\mathrm{n}=9)$. The value of RT-PCR was calculated with adjustment for the GAPDH level in $37 \mathrm{H}$ and at an RQ min/max confidence level of $95 \%(p<0.01)$. Note: The expression levels of type I collagen, osteocalcin, and VEGF were significantly higher in $\mathrm{Col} 37 \mathrm{H}$ than in $37 \mathrm{H}$. An increase in gene expression levels was noted on the $3^{\text {rd }}$ day of the culture. standard deviation. In culture assays, means were compared using an unpaired, two-tailed Student's $t$-test and significance was indicated by

${ }^{*}: \mathrm{p}<0.01,{ }^{* *}: \mathrm{p}<0.05$.

\section{Results}

\section{Evaluation of osteoblastic differentiation and VEGF on MSC}

MSC adhered to and grew normally on $37 \mathrm{H}$ and Col37H. The cell layers constructed in the tunnels were larger in Col37H (Figure 3B, 3D, $3 \mathrm{~F}$ and $3 \mathrm{H}$ ) than in $37 \mathrm{H}$ (Figure $3 \mathrm{~A}, 3 \mathrm{C}$ and $3 \mathrm{E}$ ). Thicker cells formed and larger amounts of type I collagen and VEGF were produced in the tunnels in $\mathrm{Col} 37 \mathrm{H}$ than in $37 \mathrm{H}$ after 7 days of cultivation. APasepositive cells (arrows) were observed on day 14 on Col37H (Figure 3G), and changes in APase activity were significantly higher in $\mathrm{Col} 37 \mathrm{H}$ than in $37 \mathrm{H}$ on the 7 th and $14^{\text {th }}$ days (Figure $3 \mathrm{H},{ }^{*} \mathrm{p}<0.01$ ). The expression levels of type I collagen, osteocalcin, and VEGF were significantly higher in MSC cultured with Col37H than with $37 \mathrm{H}$. These expression levels gradually increased after 3 days of cultivation (Figure 4, RQ: $\mathrm{p}<0.01)$. Furthermore, type I collagen mRNA rose 7 times as compared with contrast in day 3 and peaked in day 7 (Figure 4A). Osteocalcin mRNA was hardly detected by the contrast, but it was detected in day 3 in $\mathrm{Col} 37 \mathrm{H}$ and the expression increasing to day 14 (Figure 4B). VEGF mRNA rose 6 times as compared with contrast in day 3 , and the expression continued increasing to day 14 (Figure 4C).

\section{Quantification of bone formation}

The whole bone densities of implanted samples increased in proportion to the experimental period in $\mathrm{Col} 37 \mathrm{H}$ and $37 \mathrm{H}$ (Figure 5). Bone density was greater in $\mathrm{Col} 37 \mathrm{H}$ than in $37 \mathrm{H}$ from the first week. However, we were unable to quantify osteogenesis in each honeycomb tunnel. Therefore, we compared the bone area formed in the tunnels in each section. New bone areas increased in size in $\mathrm{Col} 37 \mathrm{H}$ and $37 \mathrm{H}$ in a time-dependent manner. The quantity of bone that formed in the honeycomb tunnels was greater in $\mathrm{Col} 37 \mathrm{H}$ than in $37 \mathrm{H}$ from the first week (Figure 6A). The bone that formed in the tunnels was longer in $37 \mathrm{H}$ than in Col37H after 4-6 weeks (Figure 6B). In contrast, the bone that formed in the tunnels was wider in Col37H than in $37 \mathrm{H}$ after 3-6 weeks (Figure 6C).

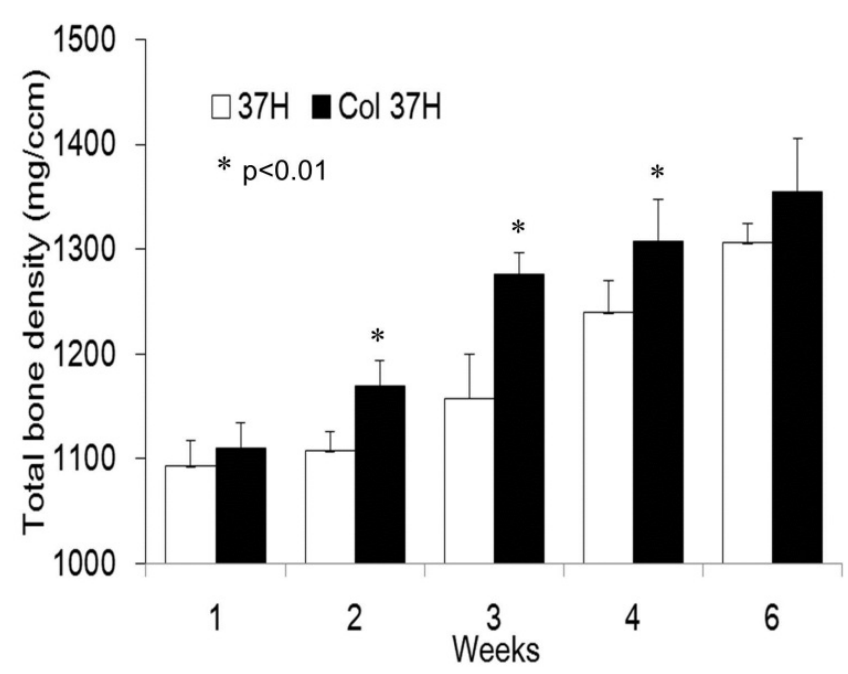

Figure 5: Quantification of whole bone densities on Col37H (black) and $37 \mathrm{H}$ (white). Total bone density was measured using the longitudinal area of ROI in each scaffold $(n=6) .{ }^{*} p<0.01$ 
Citation: Yagami K, Sadaoka S, Nakamura H, Komatsu S, Onodera J, et al. (2016) Atelocollagen Enhanced Osteogenesis in a Geometric Structured Beta-TCP Scaffold by VEGF Induction. J Tissue Sci Eng 7: 162. doi:10.4172/2157-7552.1000162

Page 5 of 8

\section{Histological evaluation of $37 \mathrm{H}$ vs. Col37H in vivo}

More well-formed soft tissue and extensive angiogenesis were observed in $\mathrm{Col} 37 \mathrm{H}$ than in $37 \mathrm{H}$ in the first week (Figures 7A and 7B). Tissue density was higher in Col37 $\mathrm{H}$ than in $37 \mathrm{H}$ in the third week, and new bone had formed in all tunnels following angiogenesis (Figures
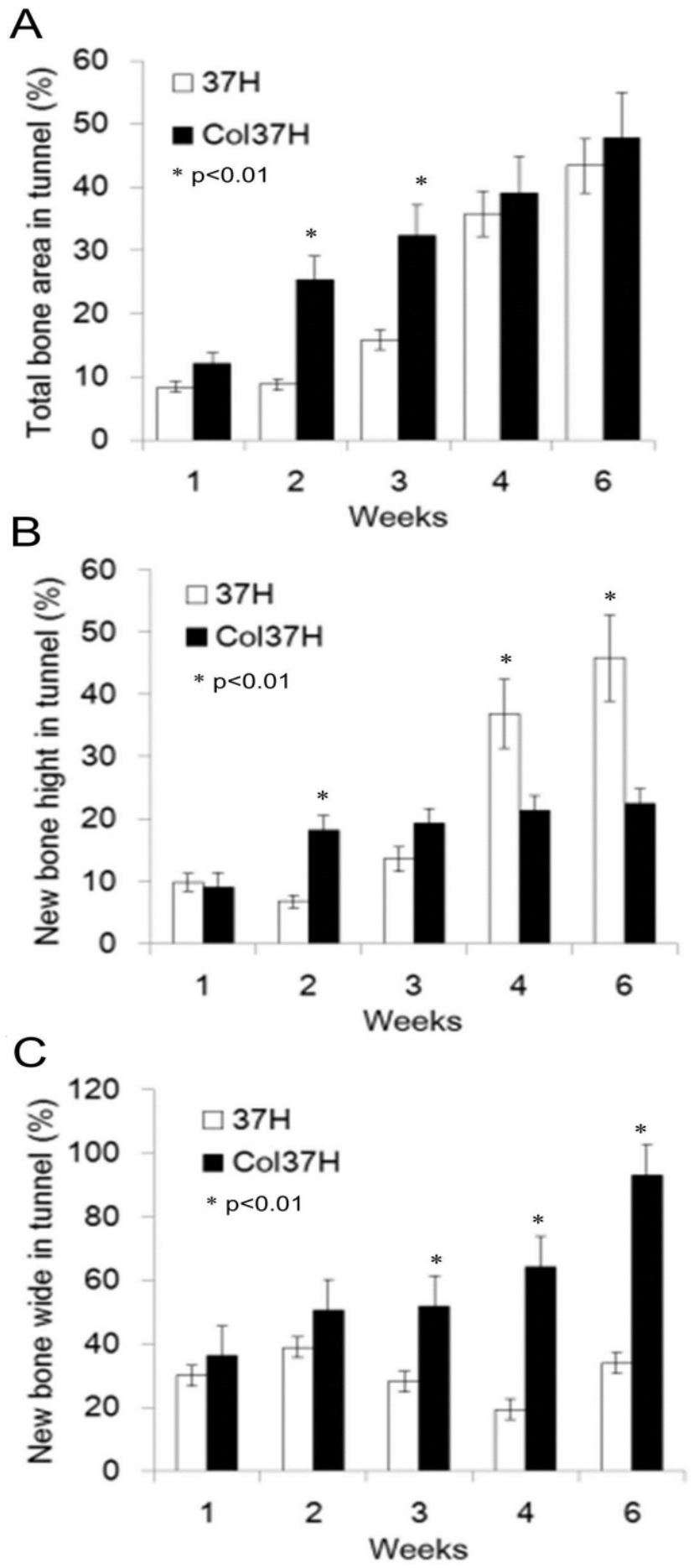

Figure 6: New bone area in Col37H and $37 \mathrm{H}$ tunnels. The total bone area (A) length $(B)$, and width $(C)$ of bones that formed in the tunnel were analyzed in undecalcified sections $(n=6)$. * $p<0.01$.
7C and 7D). The new bone penetrated the tunnels in the direction of the periosteum and continued to grow in a time-dependent manner. Maturated bone with marrow formed along the tunnel wall surface in Col37H after six weeks (Figures 7E and 7F). After 6 weeks, new bone formed directly on the inner surface of the tunnel in Col37H (Figure $7 \mathrm{~F}, \mathrm{n})$. At 1 week, VEGF was localized from the orifice to the interior of the tunnel. Capillary vessels at the entrance of the tunnel entered the interior of the tunnel (Figures $8 \mathrm{~A}$ and $8 \mathrm{~B}, \mathrm{v}$ ). Three weeks after transplantation, many capillary vessels were formed in the fibrotic tissue (f) in $\mathrm{Col} 37 \mathrm{H}$, and osteoblasts were regularly arranged on the surface of newly formed bone (Figures $8 \mathrm{C}$ and $8 \mathrm{D}, \mathrm{V}$ ).

VEGF was stained on the surface of the new bone that penetrated the tunnels as well as on blood vessels and tissues surrounding the mesenchymal tissue (Figures $8 \mathrm{C}$ and $8 \mathrm{D}$, arrow heads). Many capillary vessels containing blood cells were observed at the forefront of new bone formation.

Evaluation of changes in the expression of VEGF with osteogenesis in the presence or absence of atelocollagen

A large number of blood vessels invaded tunnels from the periosteum and existing bone surface-independent sites (Figure 9A, red). A strong VEGF signal (Figure 9B, brown) was detected around the blood vessels and the surface of the generated bone (Figure 9A, blue), which had progressed from bone. The expression of VEGF increased similarly in Col37H and 37H until after 4 weeks. The VEGF signal was stronger in $\mathrm{Col} 37 \mathrm{H}$ than in $37 \mathrm{H}$ from the first week (Figure 9C).
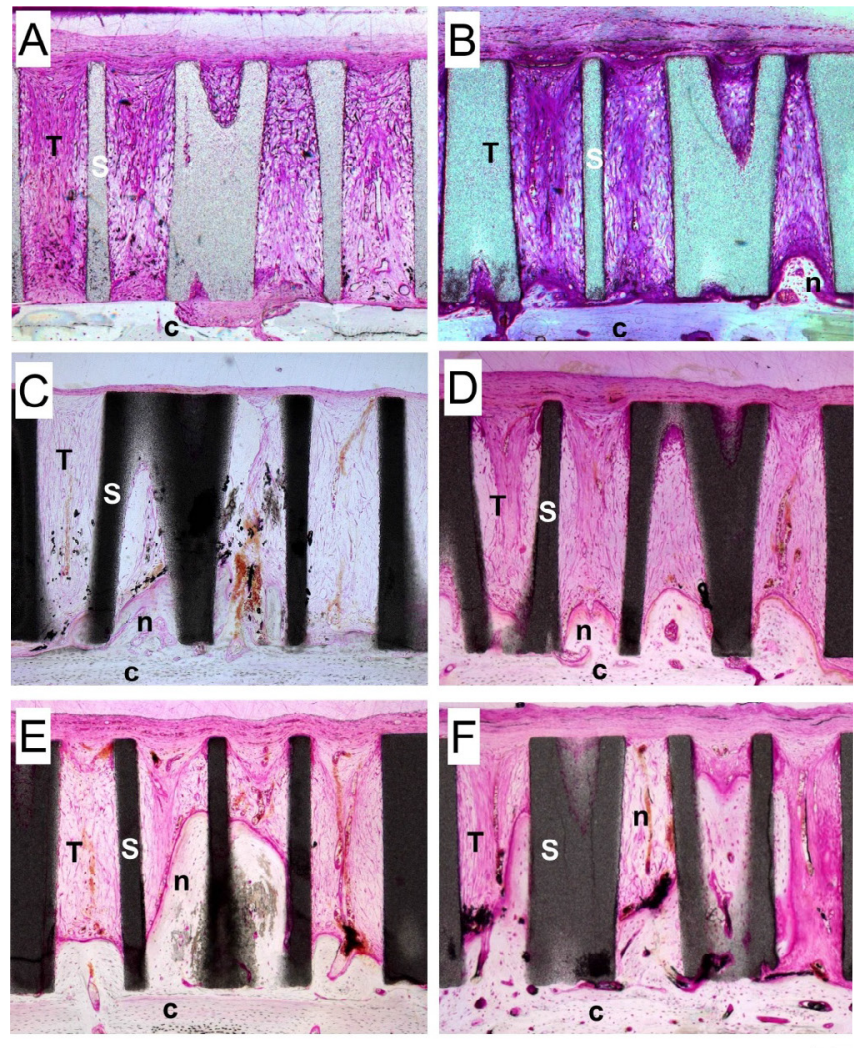

Figure 7: Histology of time-course changes in bone formation on scaffolds in the rat calvaria.37H ( $\mathrm{A}, \mathrm{C}$, and $\mathrm{E})$ and $\mathrm{Col} 37 \mathrm{H}(\mathrm{B}, \mathrm{D}$, and $\mathrm{F})$ were implanted for one (A and $B), 3(C$ and $D)$, and six (E and $F$ ) weeks. Non-decalcified sections were stained with VB. T: tunnel, S: $\beta-T C P, c$ : calvaria, n: new bone, Bar: $100 \mu \mathrm{m}$. 


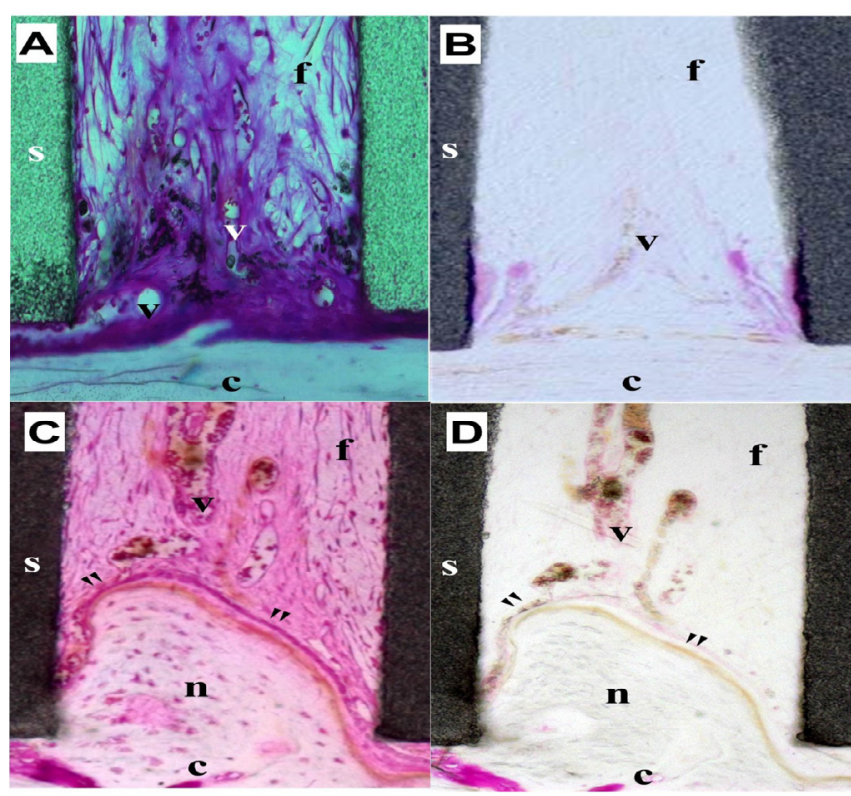

Figure 8: Microscopic findings on the relationship between blood vessels and new bone formation in a tunnel of Col $37 \mathrm{H}$. Sections were obtained 1 and 3 weeks after implantation. Undecalcified sections were stained with Villanueva bone stain ( $A$ and $C$ ), and immunological staining was performed with an anti-VEGF antibody (B and D, brown). s: $\beta-T C P, c$ : calvaria, $n$ : new bone, $v$ : vascular, f: fibrous tissue. Bar: $100 \mu \mathrm{m}$.

\section{Discussion}

We previously reported that honeycomb-shaped $\beta$-TCP $(37 \mathrm{H})$ with BMP-2-treated MSC was advantageous in terms of the initial induction of bone generation by blood vessels, which is essential for MSC to differentiate into osteoblasts. However, there is little evidence concerning the relationship between angiogenesis and the tunnel structure in the osteogenic mechanism. It currently remains unclear whether cells and blood vessels are necessary for the earlier formation of bone. Thus, we compared angiogenesis and bone generation in tunnels using VEGF. The longitudinal increase observed in the expression of VEGF supports the osteoblastic differentiation of hMSC in proportion to elevations in APase activity (Figures $3 \mathrm{G}$ and $3 \mathrm{H}$ ). Furthermore, the gene expression of VEGF in MSC was higher in Col37H than in $37 \mathrm{H}$ (Figure 3C). In the differentiation of MSC to osteoblasts, transcription factors such as Runx2 [14] and osteocalcin [13] play major roles in determining cell fate. We previously demonstrated that MSC increased the expression of Runx2, APase, and osteocalcin genes when cultured in H- $\beta$-TCP with BMP-2 [7]. In the present study, we focused on the relationship between blood vessels and osteogenesis and their effects on bone quantity with or without atelocollagen. In our in vitro experiment, we showed that the gene expression of type I collagen, osteocalcin, and VEGF by MSC was enhanced in cells cultured with Col37H (Figure 4). Furthermore, MSC formed more tubular structures along the tunnel walls of Col37H than in those of $37 \mathrm{H}$ (Figures $3 \mathrm{E}$ and $3 \mathrm{~F}$ ). These results indicate that MSC induced vascularization to maintain the base with collagen scaffold. Our results also indicated that some MSC differentiate into endothelial cells in order to construct vessels (Figures $3 \mathrm{~F}$ and 8). Kang et al. [14] found that factors other than the potential of cells were necessary for the control of ECM and growth factors in inducing angiogenesis by co-culture of BMSC and HUVEC on b-TCP. Regarding our geometric theory, the tunnel structure and presence of atelocollagen were identified as key factors of ECM for the induction and regeneration of bone.

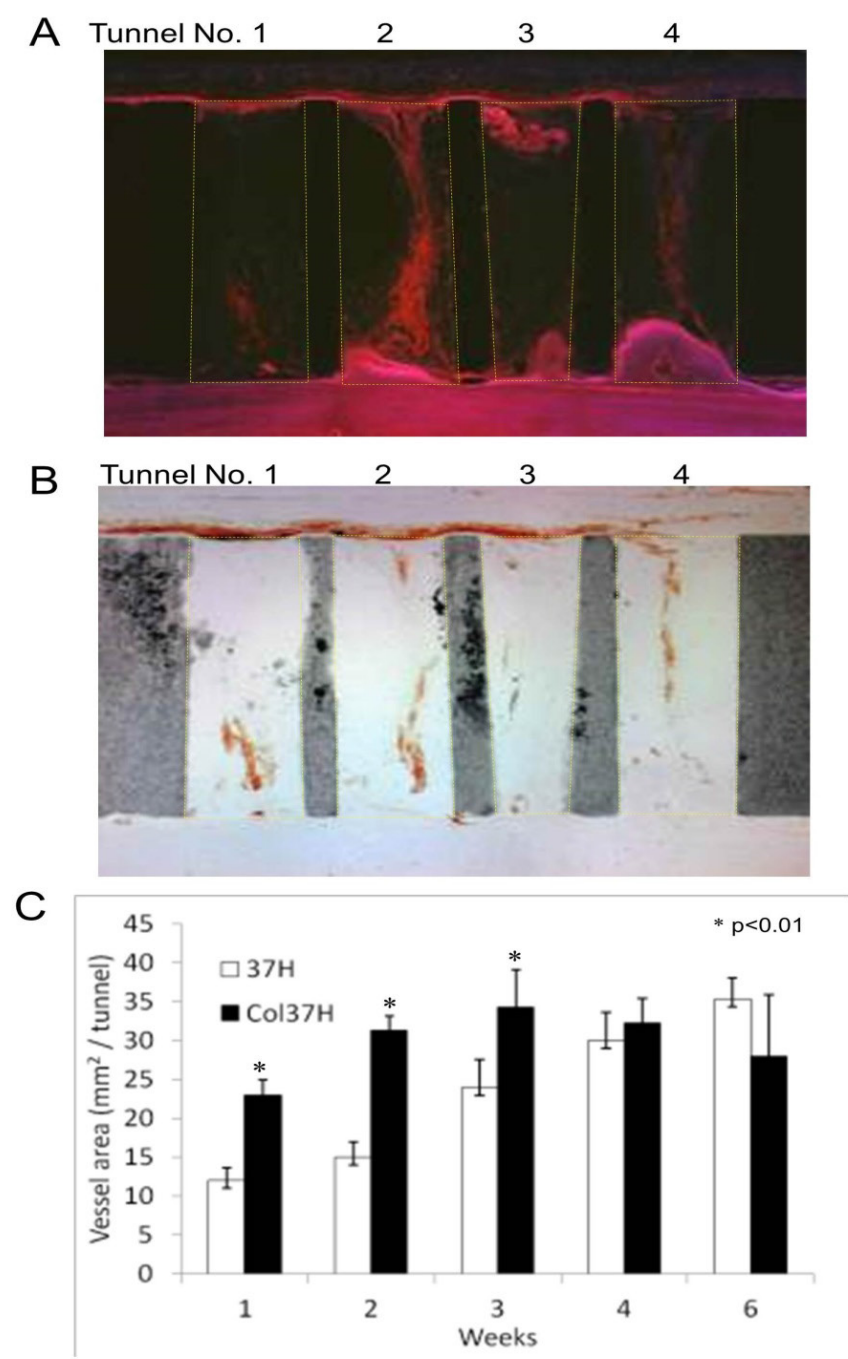

Figure 9: Evaluation of changes in the expression of VEGF with osteogenesis in the presence or absence of collagen. (A) The fluorescence microscopy photograph shows a calcein and alizarin-labeled new bone front in the tunnel. (B) A light microscope photograph showing angiogenesis in tunnels stained with VEGF on same section of $A$.

(C) A comparison ( $\mathrm{mm}^{2} /$ tunnel) of the area of angiogenesis (stained brown) per tunnel (inside of each yellow-dotted line) in $37 \mathrm{H}$ and $\mathrm{Col} 37 \mathrm{H}\left({ }^{*} \mathrm{p}<0.01\right)$.

The bone mineralization process was previously confirmed to start with the formation of nanocrystal nuclei in collagen fibrils [14]. The abilities of phosphorylated proteins, such as collagen, osteopontin, osteonectin, bone sialoprotein, and osteocalcin, to nucleate and grow calcium phosphate crystals has already been reported [15]. Growth or mineralization then occurs along the collagen fibrils, eventually interconnecting all collagen fibrils. An increase in the phosphorus concentration by matrix vesicle, epitaxial nuclei, and APase are essential for calcification [16]. The results of the present study suggested that APase produced by MSCs that differentiated into osteoblasts induced deposition of calcium phosphate in matrices such as collagen fibrils (Figures 3H and 3G). Tunnels had been filled with APase-positive cells by day 14 (Figure $3 \mathrm{~A}$ ). These results indicated that the specific structure of H- $\beta$-TCP with atelocollagen attracted a large number of MSC, and 
Citation: Yagami K, Sadaoka S, Nakamura H, Komatsu S, Onodera J, et al. (2016) Atelocollagen Enhanced Osteogenesis in a Geometric Structured Beta-TCP Scaffold by VEGF Induction. J Tissue Sci Eng 7: 162. doi:10.4172/2157-7552.1000162

Page 7 of 8

induced their differentiation and maturation to osteoblasts, thereby promoting the formation of ECM.

Maturated bone with marrow formed along the tunnel wall surfaces in $\mathrm{Col} 37 \mathrm{H}$ and $37 \mathrm{H} 3$ weeks after implantation. However, the presence or absence of atelocollagen affected some bone morphogenetic aspects. Extensive angiogenesis and increases in bone in the tunnel walls were observed earlier in Col37H than in $37 \mathrm{H}$. The local administration of atelocollagen alone without any ECM carrier did not initiate bone formation [12,17]. Our previous findings clearly showed that these carriers were not only drug delivery systems (DDS), but also functioned as an artificial ECM, on which cells grew and differentiated to form a continuous organized tissue [8]. Brkovic et al. [18] demonstrated the ability of a $\beta$-TCP-collagen material to prevent alveolar atrophy following tooth extraction in a case report.

The periosteum is indispensable to the formation of the bone However, bone regeneration in the scaffold tunnels occurred only from the existing bone side and not from the periosteal side. The periosteum is a tissue on which osteoblasts arrange on the bone surface directly under the fibrous membrane. Previous studies reported that the formation of bone on the periosteum depended on BMP-2 and VEGF [19-22]. However, even if a periosteum is not present, bone was found to form ectopically in the presence of BMP-2 [13]. Also, while pluripotent MSCs form tissues other than bone in the fracture healing process, no cartilaginous or adipose tissue was detected in this study. VEGF regulates early stage osteoblastic differentiation, and the loss of VEGF expression has been shown to decrease perichondrial vascularity and osteoblast precursor numbers [10]. Bone density was greater in $\mathrm{Col} 37 \mathrm{H}$ than in $37 \mathrm{H}$ in the third week, and bone had formed in all tunnels following angiogenesis (Figures 5 and 6). However, the presence or absence of aterocollagen affected some bone morphogenetic aspects. Extensive angiogenesis and the adherence of new bone to the tunnel wall were observed earlier in $\mathrm{Col} 37 \mathrm{H}$ than in $37 \mathrm{H}$ (Figures 7 and 8 ). Bone formation in the tunnel started from the original bone side (Figures 7-9); we previously reported that it did not form on a gingival flap side with a periosteum [8]. The distribution of VEGF expression changed to areas in agreement with the calcein- and alizarin-labeled new bone front in the tunnel (Figure 9).

The above results suggest that $\mathrm{Col} 37 \mathrm{H}$ promotes the early differentiation of host MSCs to osteoblasts by inducing angiogenesis. This method is a simple and effective technique that may be applied to the future treatment of major bone defects in clinical settings. Further investigations are necessary to clarify the reason for the occurrence of bone formation from the existing bone rather than the periosteum and the role of VEGF in this process.

\section{Conclusion}

We herein obtained novel insights into the relationship between bone regeneration and VEGF using the unique geometric properties of $\mathrm{H}-\beta$-TCP with atelocollagen in vitro and in vivo. Early vessel formation and osteogenesis were observed in $\mathrm{Col} 37 \mathrm{H}$ tunnels. These results indicate that $\mathrm{Col} 37 \mathrm{H}$ has potential in the construction of functional artificial bone.

\section{Conflict of Interest}

Kimitoshi Yagami, Sunao Sadaoka, Hiroshi Nakamura, Saho Komatsu, Jun Onodera, Masahiko Suzuki, and Yoshinori Kuboki declare that they have no conflict of interest in this study.

\section{Acknowledgement}

We thank Dr. Mariko Kimura (Pilot Corporation, Tokyo, Japan) for the fabrication of H- $\beta$-TCP. This work was supported by a Grant-in-Aid for scientific research from the Ministry of Education, Culture, Sports, Science and Technology of Japan, and a Grant-in-Aid for Scientific Research from the Japan Society for the Promotion of Science No. 19592315 (to K. Yagami).

\section{References}

1. Klijn RJ, Meijer GJ, Bronkhorst EM, Jansen JA (2010) Sinus floor augmentation surgery using autologous bone grafts from various donor sites: a meta-analysis of the total bone volume. Tissue Eng Part B Rev 16: 295-303.

2. Nakayama T, Thirukonda GJ, Nagasawa S, Kawahara I, Udagawa N, et al. (2014) Polarization of osteoclasts on dental implant materials is similar to that observed on bone. Journal of Oral Biosciences 56: 136-142.

3. Arima Y, Uemura N, Hashimoto Y, Baba S, Matsumoto N (2013) Evaluation of bone regeneration by porous alpha-tricalcium phosphate/atelocollagen sponge composite in rat calvarial defects. Orthodontic Waves 72: 23-29.

4. Sammartino G, Tia M, Gentile E, Marenzi G, Claudio PP (2009) Platelet-rich plasma and resorbable membrane for prevention of periodontal defects after deeply impacted lower third molar extraction. J Oral Maxillofac Surg 67: 23692373.

5. Tsuruga E, Takita H, Itoh H, Wakisaka $\mathrm{Y}$, Kuboki $\mathrm{Y}$ (1997) Pore size of porous hydroxyapatite as the cell-substratum controls BMP-induced osteogenesis. J Biochem 121: 317-324.

6. Kuboki Y, Iku S, Yoshimoto R, Kaku T, Takita H, et al (2009) Calcified honeycomb-shaped collagen maintains its geometry in vivo and effectively Induces vasculature and osteogenesis. Nano Biomedicine 1: 85-94.

7. Yagami K, Shirota T, Shintani S, Mayahara M, Nishizawa M, et al. (2011) Honeycomb form $\hat{~}^{2}$-tricalcium phosphate induces osteogenesis by geometrical property with BMSC. Biomed Mater Eng 21: 291-306.

8. Shirota T, Shintani S, Yoshizawa Y, Kuboki Y, Sammons R, et al. (2013) Optimal diameter of honeycomb tunnel structure induces bone regeneration and metabolism by promoting angiogenesis for an implant circumference bone defect. Journal of Hard Tissue Biology 22: 409-418.

9. Kuboki Y, Yagami K, Iku S, Kaku T, Terada M, et al. (2013) Geometry of extracellular matrix: optimal tunnel size for bone formation in disk-form honeycomb ß-TCP. NanoBiomedicine 2:107-113.

10. Duan X, Murata Y, Liu Y, Nicolae C, Olsen BR, et al. (2015) Vegfa regulates perichondrial vascularity and osteoblast differentiation in bone development Development 142: 1984-1991.

11. Ogose A, Kondo N, Umezu H, Hotta T, Kawashima H, et al. (2006) Histologica assessment in grafts of highly purified beta-tricalcium phosphate (OSferion) in human bones. Biomaterials 27: 1542-1549.

12. Kuboki Y, Jin Q, Takita H (2001) Geometry of carriers controlling phenotypic expression in BMP-induced osteogenesis and chondrogenesis. J Bone Joint Surg Am 83-83A Suppl 1: S105-15.

13. Yagami K, Uyama Y, Yoshizawa Y, Kakuta S, Yamaguchi A, et al. (2004) A human chondrogenic cell line retains multi-potency that differentiates into osteoblasts and adipocytes. Bone 34: 648-655.

14. Kang Y, Kim S, Fahrenholtz M, Khademhosseini A, Yang Y. (2013) Osteogenic and angiogenic potentials of monocultured and co-cultured human-bonemarrow-derived mesenchymal stem cells and human-umbilical-vein endothelial cells on three-dimensional porous beta-tricalcium phosphate scaffold. Acta Biomaterialia 9: 4906-4915.

15. Rhee SH, Lee JD, Tanaka T. (2000) Nucleation of hydroxyapatite crystal through chemical interaction with collagen. J Am Ceram Soc 83: 2890-2892.

16. Boskey A and Paschalis E. (2001) Matrix proteins and biomineralization, in: Bone Engineering. J Davies J, ed., EM Squared, Toronto 44-61.

17. Hessle L, Johnson KA, Anderson HC, Narisawa S, Sali A, et al. (2002) Tissue nonspecific alkaline phosphatase and plasma cell membrane glycoprotein-1 are central antagonistic regulators of bone mineralization Proc Natl Acad Sci USA 14: 9445-9449.

18. Trisi P, Rao W, Rebaudei A, Fiore P. (2003) Histologic effect of pure-phase beta-tricalcium phosphate on bone regeneration in human artificial jawbone defects. Int J Periodontics restorative Dent 23: 69-77. 
Citation: Yagami K, Sadaoka S, Nakamura H, Komatsu S, Onodera J, et al. (2016) Atelocollagen Enhanced Osteogenesis in a Geometric Structured Beta-TCP Scaffold by VEGF Induction. J Tissue Sci Eng 7: 162. doi:10.4172/2157-7552.1000162

Page 8 of 8

19. Fukui $N$, Sato $T$, Kuboki $Y$, Aoki $H$ (2008) Bone tissue reaction of nanohydroxyapatite/collagen composite at the early stage of implantation. Biomed Mater Eng 18: 25-33.

20. Yu YY, Lieu S, Lu C, Colnot C (2010) Bone morphogenetic protein 2 stimulates endochondral ossification by regulating periosteal cell fate during bone repair. Bone 47: 65-73.
21. Hoffman MD, Benoit DS. (2015) Emulating native periosteum cell population and subsequent paracrine factor production to promote tissue engineered periosteum-mediated allograft healing. Biomaterials 52: 426-440.

22. van Gastel N, Torrekens S, Roberts SJ, Moermans K, Schrooten J, et al. (2012) Engineering vascularized bone: osteogenic and proangiogenic potential of murine periosteal cells. Stem Cells 30: 2460-2471. 